\title{
Matrix and vector models in the strong coupling limit
}

\author{
D.V.Bykov, A.A.Slavnov \\ M.V.Lomonosov Moscow State University \\ Faculty of Physics, Leninskie gory, bld. 1-2, GSP-2 119992 Moscow, Russia \\ V.A.Steklov Mathematical Institute, \\ Gubkina str., bld. 8, GSP-1 119991 Moscow, Russia \\ dbykov@mi.ras.ru; slavnov@mi.ras.ru
}

\begin{abstract}
In this paper we consider matrix and vector models in the large $N$ limit $(N \times N$ matrices and vectors with $N^{2}$ components). For the case of zero-dimensional model $(\mathrm{D}=0)$ it is proved that in the strong coupling limit $g \rightarrow \infty$ statistical sums of both models coincide up to a coefficient. This is also true for $D=1$.
\end{abstract}




\section{Introduction}

Matrix models have applications in various branches of physics, but for us the main motivation will be the possibility of using them in quantum field theory. In particular, as it has been shown by 't Hooft [1], with a proper normalization of coupling constants the expansion of Green's functions (and the partition function) in $\frac{1}{N}$ corresponds in terms of ribbon Feynman diagrams to the expansion in the genuses of Riemannian surfaces, on which this graph can be drawn without self-intersections. However, even if the genus of the surface is fixed, there are infinitely many corresponding ribbon graphs and in the majority of interesting cases, unfortunately, there's still no algorithm for their summation.

The limit $N \rightarrow \infty$ is also interesting from the point of view of the Ads/CFT correspondence [2]. In this case one should consider the large $g$ limit of the sum of planar diagrams $(N \rightarrow \infty)$. This problem is far from being solved not only for gauge theories but also for the simpler case of scalar matrix models. Exceptions are cases $D=0$ and $D=1$, where the algorithm of planar diagram summation has been found [3] for real and hermitian matrix models. Later (at $D=0$ ) it was generalized for complex matrix models [4]. A review of the state-of-the-art condition of the theory of zero-dimensional matrix models can be found in [5].

Vector and matrix models at $D=0,1$ reveal remarkable similarity in the limit $g \rightarrow \infty$. The dependence of the partition function on the coupling constant in this limit is the same for vector and matrix models. This suggests that such coincidence can also take place in higher dimensions.

However, in this paper we will restrict ourselves to the consideration of a $D=0$ complex matrix model, i.e. we consider the following partition function:

$$
\mathcal{Z}(g)=\int d \varphi^{\dagger} d \varphi \exp \left[-\frac{1}{2} \operatorname{tr}\left(\varphi^{\dagger} \varphi\right)-\frac{g}{4 N} \operatorname{tr}\left(\left(\varphi^{\dagger} \varphi\right)^{2}\right)\right]
$$

where $\varphi$ is a $N \times N$ matrix. The notation $d \varphi^{\dagger} d \varphi$ should be interpreted in the following way:

$$
d \varphi^{\dagger} d \varphi \equiv D \varphi \equiv \prod_{i, j} d \operatorname{Re} \varphi_{i j} d \operatorname{Im} \varphi_{i j}
$$

Free energy is defined by

$$
\mathcal{E}=-\frac{\ln \mathcal{Z}_{B V}}{N^{2}}
$$

We will show that at $D=0$ in the strong coupling limit $(g \rightarrow \infty)$ the leading asymptotics of the free energy of this matrix model coincides with the leading asymptotics of the free energy of the corresponding vector model. At $D=1$ this is also true, but a more thorough consideration of this case is necessary. Of course, especially interesting are the cases $D \geq 2$, but there the question of whether the described hypothesis is true is still open. We would like to emphasize from the beginning that at $D \geq 2$ ultraviolet divergences appear both in matrix and vector models, therefore it only makes sense to speak about properly regularized theories.

Notice that in all dimensions linear vector models with interaction $N^{2} F\left(\frac{1}{N^{2}} \operatorname{tr}\left(\varphi^{\dagger} \varphi\right)\right)$ are exactly soluble, i.e. Green's functions of singlet variables can 
be found in explicit form, the analogy between matrix and vector models which supposedly takes place can help in the analysis of (much more complicated) matrix models.

\section{2. $\mathrm{D}=0$ bivector models}

For the comparison of matrix and vector models we first calculate the partition function of the vector model. Let's explain what we mean by vector and bivector models. The distribution $\rho$ of random variables $x_{a}(a=1 \ldots M)$ in a vector model, by definition, depends only on the square of the vector $\mathbf{x}: \rho=\rho\left(x_{a}^{2}\right)$.

Consider the partition function

$$
\mathcal{Z}_{B V}=\int D \varphi \exp \left[-\frac{1}{2} \operatorname{tr}\left(\varphi^{\dagger} \varphi\right)+N^{2} F\left(\frac{1}{N^{2}} \operatorname{tr}\left(\varphi^{\dagger} \varphi\right)\right)\right] .
$$

Although formally this model is a matrix model, in fact it is a vector model, because if we introduce a vector $y_{a}$ with $M=2 N^{2}$ components

$\left(y_{i}=\operatorname{Re} \varphi_{i, 1}, y_{N^{2}+i}=\operatorname{Im} \varphi_{i, 1}, i \leq N\right),\left(y_{i}=\operatorname{Re} \varphi_{i-N, 2}, y_{N^{2}+i}=\operatorname{Im} \varphi_{i-N, 2}, N<i \leq 2 N\right) \ldots$

then the distribution turns out to be just a function of $y_{a}^{2}$. We call such models bivector models. They are characterized by the fact that their symmetry group is much wider than in the corresponding matrix model: for example, in this case the matrix model has a symmetry group $U(1) \times S U(N)_{L} \times S U(N)_{R}$ (multiplication by a phase factor, left and right matrix multiplication $\left.{ }^{1}\right)$, whereas the vector model is invariant under $U\left(2 N^{2}\right)$. This large symmetry is what lets us find an exact solution for (bi)vector models.

First we want to find the $N \rightarrow \infty$ limit of the free energy. Inserting a unity into the integrand of (2.1) we obtain:

$$
\mathcal{Z}_{B V}=\int D \varphi d \rho \delta\left(\rho-\operatorname{tr}\left(\varphi^{\dagger} \varphi\right)\right) \exp \left[-\frac{1}{2} \operatorname{tr}\left(\varphi^{\dagger} \varphi\right)+N^{2} F\left(\frac{1}{N^{2}} \rho\right)\right] .
$$

Let us write the Fourier transformation for the delta-function:

$$
\mathcal{Z}_{B V}=(2 \pi)^{-1} \int D \varphi d \rho d \lambda \exp \left[-\frac{1}{2} \operatorname{tr}\left(\varphi^{\dagger} \varphi\right)+N^{2} F\left(\frac{1}{N^{2}} \rho\right)+i \lambda\left(\rho-\operatorname{tr}\left(\varphi^{\dagger} \varphi\right)\right)\right] .
$$

The integral over $\varphi$ is gaussian, moreover it degenerates into a product of independent integrals because the components of the matrix $\varphi$ are not linked: $\operatorname{tr}\left(\varphi^{\dagger} \varphi\right)=\sum_{i, j}\left|\varphi_{i j}\right|^{2}$. As a result, the integral over $\varphi$ can be taken, and we get:

$$
\mathcal{Z}_{B V}=(2 \pi)^{-1} \int d \rho d \lambda\left(\frac{\pi}{1 / 2+i \lambda}\right)^{N^{2}} \exp \left[N^{2} F\left(\frac{1}{N^{2}} \rho\right)+i \lambda \rho\right] .
$$

\footnotetext{
${ }^{1}$ Multiplication by a phase factor does not reduce to a combination of left and right multiplication by unimodular matrices $\varphi^{\prime}=U_{1} \varphi U_{2}^{\dagger}$. Indeed, suppose it is possible and set $\varphi=1$. Then $U_{1} U_{2}^{\dagger}=e^{i \alpha} I$, which obviously is not true if $\alpha \neq 0$.
} 
The integral over $\lambda$ can be taken in an explicit way, because we can (under the condition $\rho>0$, which follows from the definition $\left.\rho=\operatorname{tr}\left(\varphi^{\dagger} \varphi\right)\right)$ close the contour in the upper half-plane, and inside the contour there's a pole $\lambda=\frac{i}{2}$ of order $N^{2}$. The integral equals $2 \pi i \times$ (residue in this pole). Thus,

$$
\int d \lambda \frac{e^{i \lambda \rho}}{(1+2 i \lambda)^{N^{2}}}=\frac{1}{\left(N^{2}-1\right) !} \frac{2 \pi}{\rho}\left(\frac{\rho}{2}\right)^{N^{2}} e^{-\frac{\rho}{2}}
$$

We obtain

$$
\mathcal{Z}_{B V}=\frac{\pi^{N^{2}}}{\left(N^{2}-1\right) !} \int d \rho \frac{1}{\rho} \rho^{N^{2}} e^{N^{2} F\left(\frac{\rho}{N^{2}}\right)-\frac{\rho}{2}} .
$$

Next we make a change $\rho \rightarrow N^{2} \rho$ to reduce the integral to the case, in which the steepest descent method is applicable:

$$
\mathcal{Z}_{B V}=\frac{\left(N^{2} \pi\right)^{N^{2}}}{\left(N^{2}-1\right) !} \int d \rho \frac{1}{\rho} e^{N^{2}\left(\ln \rho+F(\rho)-\frac{\rho}{2}\right)} .
$$

The stationary point equation is

$$
\rho_{0}: \frac{1}{\rho_{0}}-\frac{1}{2}+F^{\prime}\left(\rho_{0}\right)=0 \text {. }
$$

Now we specify the potential: $F(\rho)=-\frac{g}{4} \rho^{2}$. Then the equation takes the form

$$
\frac{1}{\rho_{0}}-\frac{1}{2}-\frac{g}{2} \rho_{0}=0
$$

A positive solution of the equation is

$$
\rho_{0}=\frac{1}{2 g}(\sqrt{1+8 g}-1)
$$

We get the following expression for the partition function in the large $N$ limit:

$$
\mathcal{Z}_{B V}=\frac{\left(N^{2} \pi\right)^{N^{2}}}{\left(N^{2}-1\right) !} \frac{1}{\rho_{0}}\left(\frac{2 \pi}{N^{2} g\left(\frac{1}{2}+\frac{1}{2-\rho_{0}}\right)}\right)^{1 / 2} e^{N^{2}\left(\ln \rho_{0}-\frac{\rho_{0}}{2}-g \frac{\rho_{0}^{2}}{4}\right)}
$$

Using Stirling's formula for the factorial $\left(n ! \approx\left(\frac{n}{e}\right)^{n} \sqrt{2 \pi n}\right)$,

$$
\mathcal{Z}_{B V}=(e \cdot \pi)^{N^{2}} \frac{1}{\rho_{0}}\left(\frac{g}{2}+\frac{g}{2-\rho_{0}}\right)^{-1 / 2} e^{N^{2}\left(\ln \rho_{0}-\frac{\rho_{0}}{2}-g \frac{\rho_{0}^{2}}{4}\right)}
$$

From (2.10) it is clear that in the limit $g \rightarrow \infty: \rho_{0} \sim\left(\frac{2}{g}\right)^{1 / 2} \rightarrow 0$. Therefore the only growing contribution to the free energy is given by the logarithm, and

$$
\mathcal{E}_{0} \underset{g \rightarrow \infty}{\rightarrow} \frac{1}{2} \ln (g)
$$


3. Solution of the $\mathrm{D}=0$ matrix model with $\operatorname{tr}\left(\left(\varphi^{\dagger} \varphi\right)^{2}\right)$-interaction

In this section we consider a model which has the $N \times N$ complex matrix $\varphi$ as its dynamical variable. We will consider the partition function

$$
\mathcal{Z}=\int d \varphi^{\dagger} d \varphi \exp \left[-\frac{1}{2} \operatorname{tr}\left(\varphi^{\dagger} \varphi\right)-\frac{g}{4 N} \operatorname{tr}\left(\left(\varphi^{\dagger} \varphi\right)^{2}\right)\right] .
$$

Correlation functions of operators $\operatorname{tr}\left(\left(\varphi^{\dagger} \varphi\right)^{m}\right)$ were obtained in [4]. All planar Green's functions are constructed with the help of the eigenvalue distribution function $u(\lambda)$, defined on a segment $\lambda \in[0 ; \sqrt{z}$ (here $\lambda$ should be interpreted as the modulus of an eigenvalue, so $\lambda \geq 0$ ), which has the following form:

$$
u(\lambda)=\frac{1}{2 \pi}\left(1+\frac{g z}{2}+g \lambda^{2}\right) \sqrt{z-\lambda^{2}},
$$

where $z=\frac{2}{3 g}(\sqrt{1+24 g}-1)$. Note that as $g \rightarrow \infty z \rightarrow 0$. At the same time the area under the plot $u(\lambda)$ is always equal to unity: $\int u(\lambda) d \lambda=1$. As a result, obviously

$$
\lim _{g \rightarrow \infty} u(\lambda)=\delta(\lambda)
$$

where the limit should be understood, of course, in the sense of distributions. One can see that in the limit $g \rightarrow \infty$ all eigenvalues tend to approach zero. Nevertheless, it's not possible to use directly the asymptotics (3.3) in order to obtain the partition function at large $g$, as in the expression for the free energy there's a term $\int d \lambda u(\lambda) \ln \lambda$, and the logarithm is singular at $\lambda=0$. We propose another method for the solution of a complex matrix model, which is free from the mentioned drawback, and, using this method, we will prove at $D=0$ the statement presented above about the equality of free energies. First we make in the integral the Hubbard-Stratonovich transformation of the second term in the exponent:

$$
\mathcal{Z}=\int d \varphi^{\dagger} d \varphi d \eta \exp \left[-\frac{1}{2} \operatorname{tr}\left(\varphi^{\dagger} \varphi\right)-\operatorname{tr} \eta^{2}+i \sqrt{\frac{g}{N}} \operatorname{tr}\left(\eta \varphi^{\dagger} \varphi\right)\right],
$$

where $\eta$ is a hermitian matrix. Now we make the gaussian integration over $\varphi$ :

$$
\mathcal{Z}=\int d \eta \exp \left[-\operatorname{tr} \eta^{2}-N \operatorname{tr} \ln \left[\frac{1}{2} I-i \sqrt{\frac{g}{N}} \eta\right]\right] .
$$

After passing to the integration over (real) eigenvalues $\lambda_{k}$ of a hermitian matrix $\eta$ the integral can be calculated by the stationary phase method. Upon varying the exponent over $\lambda_{k}$ we get the following equations:

$$
\sum_{j=1}^{\prime} \frac{2}{\lambda_{k}-\lambda_{j}}=2 \lambda_{k}+\frac{i \sqrt{g N}}{-1 / 2+i \sqrt{\frac{g}{N}} \lambda_{k}}, k=1 \ldots N .
$$

In spite of the fact that we're integrating over real space $\mathbb{R}^{N}$, the values $\lambda_{k}$ in the 
stationary point in our case are complex - it is a usual situation for the steepest descent method. Let's now pass to the continuous case $(N \rightarrow \infty)$, changing $\lambda_{k} \rightarrow$ $\sqrt{N} \lambda(x)(x \in[0,1]$ is an analog of the index $k$ for the continuous case). Then we get a system

$$
\begin{aligned}
\mathrm{P} \int_{C} \frac{v(\nu) d \nu}{\lambda-\nu} & =\lambda+\frac{\sqrt{g}}{2} \frac{1}{\sqrt{g} \lambda+\frac{i}{2}} \\
\int_{C} v(\nu) d \nu & =1,
\end{aligned}
$$

where $v(\lambda)=\frac{d x}{d \lambda}$. From the discrete form (3.6) of our equation one can see that, if $\left\{\lambda_{k}\right\}_{k=1 \ldots N}$ is a solution, so is $\left\{-\lambda_{k}^{*}\right\}_{k=1 \ldots N}$, therefore the contour $C$ is symmetric with respect to the axis $O y=\operatorname{Im} \lambda$. Next we multiply the first equation by $1-2 i \sqrt{g} \lambda$ and introduce a new function $\bar{v}(\lambda)=(1-2 i \sqrt{g} \lambda) v(\lambda)$. According to the position of the contour, $\int_{C} \bar{v}(\lambda) d \lambda=D$ is real (because $\operatorname{tr} \lambda=\int_{C} \lambda u(\lambda) d \lambda$ is imaginary). Then we get an equation

$$
\mathrm{P} \int_{C} \frac{\bar{v}(\nu) d \nu}{\lambda-\nu}=i \sqrt{g}+\lambda(1-2 i \sqrt{g} \lambda)
$$

We will look for a "resolvent" $\Phi(\lambda) \equiv \int_{C} \frac{\bar{v}(\nu) d \nu}{\lambda-\nu}, \lambda \notin C$ in the following form

$$
\Phi(\lambda)=i \sqrt{g}+\lambda(1-2 i \sqrt{g} \lambda)+(A \lambda+B) \sqrt{\left(\lambda+b^{*}\right)(\lambda-b)}
$$

In the limit $\lambda \rightarrow \infty$ the asymptotics is known $\Phi(\lambda) \rightarrow \frac{D}{\lambda}$ (which is clear from the definition of the resolvent and the normalization condition - see above), but since $D$ is unknown we will use the following conditions: the coefficients of $\lambda^{2}, \lambda^{1}, \lambda^{0}$ vanish and $\int_{C} v(\nu) d \nu=\int_{C} \frac{\bar{v}(\nu) d \nu}{1-2 i \sqrt{g} \nu}=1$. The first three conditions can be rewritten in the following form:

$$
\begin{aligned}
& \lambda^{2}:-2 i \sqrt{g}+A=0 \\
& \lambda^{1}: 1+B+i \sqrt{g}\left(b^{*}-b\right)=0 \\
& \lambda^{0}: \sqrt{g}(\operatorname{Re} b)^{2}+B \operatorname{Im} b-\sqrt{g}=0
\end{aligned}
$$

In writing out these equations we took the positive value of the square root on the continuation of the segment $\left[-b^{*} ; b\right]$ to the right of $b$. Then on the axis, which is perpendicular to this segment and intersects it in the middle, the square root is imaginary with a positive (above the intersection point) or negative (below the intersection point) imaginary part. Suppose $\lambda_{0} \in C$. Taking into account what has been said and the Sokhotsky-Plemelj formulas,

$$
\lim _{\epsilon \rightarrow 0}\left(\Phi\left(\lambda_{0}+i \epsilon\right)-\Phi\left(\lambda_{0}-i \epsilon\right)\right)=2 i\left(A \lambda_{0}+B\right) \sqrt{\left(\lambda_{0}+b^{*}\right)\left(b-\lambda_{0}\right)}=-2 i \pi \bar{v}\left(\lambda_{0}\right),
$$


thus

$$
\bar{v}(\lambda)=-\frac{1}{\pi}(A \lambda+B) \sqrt{\left(\lambda+b^{*}\right)(b-\lambda)}, \lambda \in C .
$$

Therefore we can rewrite the last condition in the form

$$
-\frac{1}{\pi} \int_{-b^{*}}^{b} \frac{A \nu+B}{1-2 i \sqrt{g} \nu} \sqrt{\left(\nu+b^{*}\right)(b-\nu)} d \nu=1 .
$$

So we're interested in the integrals of the following type:

$$
\begin{aligned}
& I_{1}=\int_{-b^{*}}^{b} \sqrt{\left(\nu+b^{*}\right)(b-\nu)} d \nu \\
& I_{2}=\int_{-b^{*}}^{b} \frac{d \nu}{1-2 i \sqrt{g} \nu} \sqrt{\left(\nu+b^{*}\right)(b-\nu)} .
\end{aligned}
$$

They can be calculated in a similar fashion. Actually these integrals are contour integrals along the upper bank of the cut from left to right. Since on the lower bank of the cut the integrand takes on an opposite value, the value of our integral is half the value of the integral along a contour closed clockwise. The integral along a closed contour in the first case $I_{1}$ is just $2 \pi i \times$ (residue at infinity), and in the second case $I_{2}$ one also has to take into account the residue at the pole. Adopting the notation $\tau(\nu)=\sqrt{\left(\nu+b^{*}\right)(b-\nu)}$,

$$
\begin{aligned}
& I_{1}=\pi \operatorname{res}(\tau(\nu), \infty) \\
& I_{2}=\pi\left(\operatorname{res}\left(\frac{\tau(\nu)}{1-2 i \sqrt{g} \nu}, \infty\right)+\operatorname{res}\left(\frac{\tau(\nu)}{1-2 i \sqrt{g} \nu}, \nu_{0}\right)\right),
\end{aligned}
$$

were $\nu_{0}=\frac{1}{2 i \sqrt{g}}$ is the position of the pole. Upon calculating we obtain:

$$
\begin{gathered}
I_{1}=\frac{\pi}{2}(\operatorname{Re} b)^{2} \\
I_{2}=\frac{\pi}{2 \sqrt{g}}\left[\left((\operatorname{Re} b)^{2}+\left(\operatorname{Im} b+\frac{1}{2 \sqrt{g}}\right)^{2}\right)^{1 / 2}-\operatorname{Im} b-\frac{1}{2 \sqrt{g}}\right] .
\end{gathered}
$$

Note that from the definition of the integral $I_{2}(g)$ it is clear that it is nonsingular as $g \rightarrow 0$. Formally there are singularities in the explicit expression obtained for $I_{2}$, but it is easy to see that they cancel each other. So it is a check of our calculations. 
Let's now write out all the conditions on $(B, \operatorname{Re} b, \operatorname{Im} b)$, eliminating $A$ :

$$
\begin{aligned}
B & =-1-2 \sqrt{g} \operatorname{Im} b \\
0 & =\sqrt{g}(\operatorname{Re} b)^{2}+B \operatorname{Im} b-\sqrt{g} \\
1 & =\frac{1}{\pi} I_{1}+\frac{2 \sqrt{g} \operatorname{Im} b}{\pi} I_{2}
\end{aligned}
$$

The equations are real, hereupon the number of unknowns coincides with the number of equations. This confirms that it was quite relevant to take into account the symmetry of the contour. Next we introduce the parameter $\varepsilon=\frac{1}{2 \sqrt{g}}$ and eliminate $B$ :

$$
\begin{aligned}
\frac{1}{2} & =\frac{1}{2}(\operatorname{Re} b)^{2}-(\operatorname{Im} b)^{2}-\varepsilon \operatorname{Im} b \\
1 & =\frac{1}{2}(\operatorname{Re} b)^{2}-(\operatorname{Im} b)^{2}-\varepsilon \operatorname{Im} b+\operatorname{Im} b \sqrt{(\operatorname{Re} b)^{2}+(\operatorname{Im} b+\varepsilon)^{2}} .
\end{aligned}
$$

Passing to the limit $\varepsilon \rightarrow 0$, introducing $\bar{b}=\lim _{\varepsilon \rightarrow 0} b(\varepsilon)$ and eliminating $(\operatorname{Re} b)^{2}$ with the help of the first equation, we get

$$
\frac{1}{2}=\operatorname{Im} \bar{b} \sqrt{3(\operatorname{Im} \bar{b})^{2}+1}
$$

The solution is as follows:

$$
\operatorname{Im} \bar{b}=\frac{1}{\sqrt{6}} ; \quad \operatorname{Re} \bar{b}=\frac{2}{\sqrt{3}} .
$$

Thus, the contour $C$ lies above the real axis, so the pole $\nu_{0}=-i \varepsilon$ never hits the contour, as one could expect. The ends of the contour in the limit $\epsilon \rightarrow 0$ turned out to be on a finite distance from each other as well as from the origin. It means that this approach correctly reflects the asymptotic properties of the model - "collapsing" of the contour, which took place in (3.3) as $g \rightarrow \infty$, is absent. Let's now return to the problem of calculating the partition function. As it is clear from (3.5), the free energy in the limit $N \rightarrow \infty$ is:

$$
\mathcal{E}=\int d \lambda v(\lambda)\left(\lambda^{2}+\ln \left(\frac{1}{2}-i \sqrt{g} \lambda\right)\right)-\int d \lambda d \mu v(\lambda) v(\mu) \ln |\lambda-\mu| .
$$

From the presented results one can see that there's a finite limit of the function $v(\lambda)$, as $g \rightarrow \infty$. Therefore, the main contribution to $\mathcal{E}$ in the described limit is given by $\sqrt{g}$, which appears under the first logarithm, thus

$$
\mathcal{E} \underset{g \rightarrow \infty}{\rightarrow} \frac{1}{2} \ln g
$$

which exactly coincides with the analogous expression for the vector model (2.13). 


\section{Discussion and conclusion}

In this paper we have presented a detailed consideration of the $D=0$ complex model. In particular, we obtained an expression for the partition function by a method, analogous to the method presented in [3]. To the leading order in $g$ in the strong coupling limit the hypothesis about the analogy of the matrix and vector models turns out to be true. Moreover, it is also true in the next-to-leading order if one uses in the vector model a finitely renormalized charge $\widetilde{g}=\alpha \cdot g$ ( $\alpha=$ const.) instead of $g$. This is clear from the general structure of the free energy for both models:

$$
E=\frac{1}{2} \ln g+\beta_{0}+\frac{\beta_{1}}{\sqrt{g}}+\ldots
$$

We can always set the constant $\beta_{0}$ to any value by changing in an appropriate way $g \rightarrow \alpha \cdot g$. What is nontrivial is the fact that the functional structures in the strong coupling expansion of the matrix and vector models coincide, although the coefficients may differ (a few first coefficients can be made equal by an appropriate finite renormalization of parameters).

Our hypothesis is also true at $D=1$, which can be seen from the explicit form of the free energy asymptotics for the matrix and vector models: both behave like

$E \sim \kappa \cdot g^{1 / 3}(\kappa=$ const $)$. The question of whether our hypothesis is true for $D \geq 2$ remains open.

\section{Acknowledgments}

Our work was partially financed by the Grant for the Support of Leading Scientific Schools NSch-672.2006.1, the RFBR grant 05-01-00541. D.B. is grateful to Prof. M.Müller-Preußker for hospitality at the Humboldt University of Berlin, where a part of this work was done, to the German Society for Academic Exchanges (DAAD) for financial support in the framework of a Leonard Euler scholarship and to the "Dynasty" foundation for financial support.

\section{References}

[1] G. 't Hooft "Planar diagram field theories", Progress in gauge field theory, NATO Adv. Study Inst. Series (1984)

[2] O.Aharony, S.S.Gubser, J.Maldacena, H.Ooguri, Y.Oz "Large N Field Theories, String Theory and Gravity", Phys.Rept. 323 (2000) 183-386, hep-th/9905111

[3] E. Brezin, C. Itzykson, G. Parisi, J.B. Zuber "Planar diagrams", Commun. math. Phys. 59, 35-51 (1978)

[4] J.Ambjorn, J.Jurkiewicz, Yu.M.Makeenko "Multiloop correlators for two-dimensional quantum gravity", Phys. Lett. B, v.251 4 p.517 (1990)

[5] L.O.Chekhov, "Matrix models with hard walls: geometry and solutions", hep-th/0602013 